\title{
Effect of Fermentation Time of Kombucha Tea on Its Hypoglycaemic Activity in Rats
}

\author{
Nur A. Setiani, Rika L. Anggriani, Anggi Restiasari \\ Indonesia School of Pharmacy, Bandung, West Java, Indonesia
}

\begin{abstract}
Diabetes mellitus is a chronic metabolic disorder characterized by hyperglycaemia. Functional food, such as kombucha tea, is widely used as complementary treatment for type 2 diabetes mellitus. Kombucha tea is made through fermentation process of green or black tea using a microbial kombucha consortium (Acetobacter xylinum and several types of yeast). The aim of this study was to evaluate the effect of fermentation time of kombucha tea on its hypoglycaemic activity in rats. The green tea (Camellia sinensis) was fermented with kombucha consortium for 8, 14, and 21 days. Evaluation of hypoglycaemic activity was conducted using glucose tolerance test method. First, fasting blood glucose levels in rats were measured after 16 hours fasting. Hyperglycaemic condition was induced by administering glucose $2 \mathrm{~g} / 0.2 \mathrm{~kg}$ body weight. Blood glucose levels were measured again after 30 minutes. Subsequently, $5.5 \mathrm{ml}$ of kombucha tea in various fermentation time was orally administered. Blood glucose levels were measured at 30, 60, 90, 120, 150 and 180 minutes after kombucha tea administration. The results revealed the average reduction of blood glucose were $18.16 \%, 33.64 \%$, and $19.88 \%$ by kombucha tea fermented for 8,14 , and 21 days, respectively. In conclusion, kombucha tea fermented for 14 days is potential to be developed as a hypoglycaemic agent.
\end{abstract}

Keywords: fermentation time, glucose level, hypoglycaemia, kombucha tea

\section{Introduction}

Hyperglycaemia is a medical condition that is characterized by elevated blood glucose levels. It is the main symptom of diabetes mellitus, a metabolic disorder associated with insulin deficiency and insulin resistance. The cornerstone of diabetes treatment is healthy diet, regular physical activity, and pharmacological treatment, such as oral hypoglycamic drugs and if necessary, insulin. ${ }^{1}$
Functional food can be an alternative to complement a healthy lifestyle. It is defined as a food (not medicine or supplement) which naturally or has been through the process, contains bioactive compounds that provide health benefits. One of the functional foods is kombucha tea. It is a beverage made from green or black tea which is fermented using microbial kombucha consortium. ${ }^{2}$ Kombucha tea has a slightly acidic taste and contains

Corresponding author: Nur A. Setiani. Indonesia School of Pharmacy, Bandung, West Java, Indonesia.

Email: nur.asni@stfi.ac.id

Received: 10 February 2019. Revised: 26 March 2019. Published: 25 April 2019. 
less alcohol as a result of the fermentation process. ${ }^{3}$ In the initial process of fermentation, yeast culture in kombucha will convert sucrose to glucose and fructose for ethanol production. Acetic acid is produced from fructose, while glucuronic acid is made from glucose. Various other organic acids are also generated. ${ }^{4,5}$ Glucuronic acid, lactic acid, vitamins, and other compunds make kombucha tea have many health benefits. ${ }^{5,6}$

Nevertheless, there are limited studies regarding the effect of fermentation time of kombucha tea on its hypoglycaemic activity. The aim of this study was to evaluate the effect of fermentation time of kombucha tea on its hypoglycaemic activity in rats. Determination of kombucha dose is based on safe doses of kombucha tea consumed by humans. $^{7}$

\section{Methods}

Tea leaves (Camellia sinensis) were derived from Gambung, Ciwidey, West Java, Indonesia. Plant determination was conducted at Jatinangor Herbarium, Laboratory of Plant Taxonomy, Department of Biology, Faculty of Mathematics and Natural Sciences, Padjadjaran University, Bandung. Examination of the characteristics of the tea leaves include total ash content, water content, ethanol soluble content, and water solube content. Phytochemical screening was also performed to determine the presence of secondary metabolites, such as alkaloids, tannins, flavonoids, monoterpenoids and sesquiterpenoids, triterpenoids, and saponins. Kombucha tea was made by adding 15 grams of green tea and $100 \mathrm{~g}$ of sugar to 11 of boiling water. Green tea solution was put at room temperature $\left(20-30^{\circ} \mathrm{C}\right)$ for 15 minutes before it was filtered. Kombucha consortium culture was then added to the green tea. Incubation process was done at room temperature (20$30^{\circ} \mathrm{C}$ ) for 8,14 and 21 days.

The experimental animals consisted of 18 male Wistar strain white rats. The rats were conditioned for 1 week and divided into 6 groups, i.e., normal control (did not receive hyperglycaemic induction and kombucha treatment); negative control (received hyperglycaemic induction, but did not receive kombuca treatment); positive control (received hyperglycaemic induction and oral hypoglycaemic drugs, i.e., glibenclamide); and 3 treatment groups (received hyperglycaemic induction and kombucha fermented at 8,14, and 21 days). Measurement of blood glucose levels was carried out after 16 hours of fasting. Hyperglycaemic condition was induced by administering 10 $\mathrm{g} / \mathrm{kg}$ or equivalent to $2 \mathrm{~g} / 0.2 \mathrm{~kg}$ body weight of glucose in groups 2, 3, 4, 5 and 6. Blood glucose levels were measured again after 30 minutes. Subsequently, $5.5 \mathrm{ml}$ of kombucha tea in various fermentation time was orally administered. Blood glucose levels were measured at 30,60,90,120,150 and 180 minutes after kombucha tea administration. ${ }^{6}$ Measurement of glucose level was conducted using the Easy Touch ${ }^{\circledR}$ glucometer tool.

\section{Results and Discussion}

Plant determination showed that the plant used in this study was Camellia sinensis

Table 1. Characterization of Tea Leaves (Camellia sinensis)

\begin{tabular}{lcc}
\hline \multicolumn{1}{c}{ Characteristic } & Result (\%) & MMI Standard \\
\hline Total ash content & 4.5 & $<7 \%$ \\
Ethanol soluble content & 32.0 & $>9 \%$ \\
Water soluble content & 31.0 & $>8 \%$ \\
Water content & 3.5 & - \\
\hline
\end{tabular}


Table 2. Phytochemical Screening of Tea Leaves (Camellia sinensis)

\begin{tabular}{lc}
\hline \multicolumn{1}{c}{ Chemical compound } & Screening \\
\hline Alkaloid & + \\
Phenolic & + \\
Tanin & + \\
Flavonoid & + \\
Monoterpen dan sesquiterpen & + \\
Steroid dan triterpenoid & - \\
Quinon & + \\
Saponin & + \\
\hline
\end{tabular}

$+=$ Detected, $-=$ Not Detected

(L.) Kuntze. Characterization of tea leaves was conducted to determine the feasibility of green tea leaves for kombucha tea production. Table 1 shows that characteristics of the sample was in accordance with the Materia Medica Indonesia (MMI) requirements. Phytochemical screening was performed to identify metabolites in tea leaves. We found the presence of phenolic and flavonoids (Table 2) that may play a role in its hypoglycaemic activity. ${ }^{8}$

The blood glucose level before and afer glucose induction can be seen in the Table 3. Fasting blood glucose level was less than than $100 \mathrm{mg} / \mathrm{dl}$, indicating normal blood glucose level. After induction, there was an increase in blood glucose level, with the average increase of $130.132 \mathrm{mg} / \mathrm{dl}$. Table 4 shows blood glucose level after kombucha tea ttreatment. Based on analysis of variance (ANOVA), we found that the treatment group has different hypoglycaemic effect compared to control. The similar effect was observed in group treated with kombucha tea which is fermented for 8 and 14 days with the positive control (treated with glibenclamide).

Figure 1 shows the reduction of blood glucose levels in all groups. We found that the percentage of reduction were $18.16 \%, 33.64 \%$, and $19.88 \%$, for kombucha tea feremented at 8,14 , and 21 days, respectively. The result indicated that kombucha tea fermented for 14 days is potential hypoglycaemic agent. It is estimated that hypoglycaemic activity

Table 3. Average of Glucose Levels Before and After Hyperglycaemia Induction

\begin{tabular}{cccc}
\hline \multirow{2}{*}{ No } & \multirow{2}{*}{ Group } & \multicolumn{2}{c}{ Glucose levels (mg/d) } \\
\cline { 3 - 4 } & & Normal & Induced \\
\hline 1 & $\mathrm{KN}$ & $96.00 \pm 3.27$ & - \\
2 & $\mathrm{~K}(+)$ & $92.33 \pm 6.65$ & $138.00 \pm 4.92$ \\
3 & $\mathrm{~K}(-)$ & $89.33 \pm 5.25$ & $128.33 \pm 5.88$ \\
4 & $\mathrm{P} 1$ & $88.33 \pm 2.50$ & $123.00 \pm 11.51$ \\
5 & $\mathrm{P} 2$ & $92.33 \pm 4.10$ & $139.00 \pm 5.35$ \\
6 & $\mathrm{P} 3$ & $88.00 \pm 7.25$ & $122.33 \pm 11.61$ \\
\hline
\end{tabular}

$\mathrm{KN}=$ normal control, $\mathrm{K}(+)=$ positive control, $\mathrm{K}(-)=$ negative control, $\mathrm{P} 1$ = Kombucha fermented for 8 days, $\mathrm{P} 2=$ Kombucha fermented for 14 days, $\mathrm{P} 3=$ Kombucha fermented for 21 days 
Table 4. Average of Glucose Levels after Treatment

\begin{tabular}{|c|c|c|c|c|c|c|c|c|}
\hline \multirow{2}{*}{ No } & \multirow{2}{*}{ Group } & \multicolumn{6}{|c|}{ Sampling time (minutes) } & \multirow{2}{*}{ Average } \\
\hline & & 30 & 60 & 90 & 120 & 150 & 180 & \\
\hline 1 & $\mathrm{KN}$ & 97.67 & 93.54 & 88 & 87.66 & 85.67 & 94.11 & $91.10 \pm 4.26$ \\
\hline 2 & $\mathrm{~K}(+)$ & 123.33 & 118.18 & 112.66 & 107.1 & 100.66 & 93.67 & $109.26 \pm 9.34$ \\
\hline 3 & $\mathrm{~K}(-)$ & 132.12 & 128.67 & 123.67 & 120.09 & 112.33 & 107 & $120.64 \pm 8.76$ \\
\hline 4 & $\mathrm{P} 1$ & 125 & 125.33 & 117 & 112.67 & 107.33 & 100.66 & $114.66 \pm 8.82$ \\
\hline 5 & $\mathrm{P} 2$ & 134 & 128 & 120.66 & 112.34 & 99 & 92.24 & $114.37 \pm 14.94$ \\
\hline 6 & P3 & 128.66 & 126.33 & 130 & 132 & 116.33 & 98 & $121.88 \pm 11.79$ \\
\hline
\end{tabular}

of kombucha tea is caused by phenolic compounds and gluconic acid produced after the fermentation process. ${ }^{9}$ These high antioxidant compunds may help regeneration of pancreatic $\beta$ cells that can improve insulin secretion. ${ }^{6,89}$ Another study reported that phenolic compound and organic acid could also decrease the absorption of glucose from the digestive system and provide inhibitory effects on the activity of $\alpha$-amylase. ${ }^{1,10}$

The differences in the reduction of blood glucose levels is caused by differences in the levels of antioxidant and gluconic acid based on fermentation time. Microbial kombucha consortium breaks down the sugar into glucose and fructose. Glucose is used to produce alcohol and carbon dioxide. It is also metabolized to glucuronic and acetic acid by the activity of Acetobacter, while fructose is still used by microorganisms in small amounts. Our finding is similar with previous study showing the content of glucuronic acid increased during fermentation with the optimal time of 10 days. ${ }^{11}$

\section{Conclusion}

Kombucha tea fermented for 14 days is potential to be developed as a hypoglycaemic agent.

\section{Acknowledgemment}

None declared.

\section{Funding}

None.

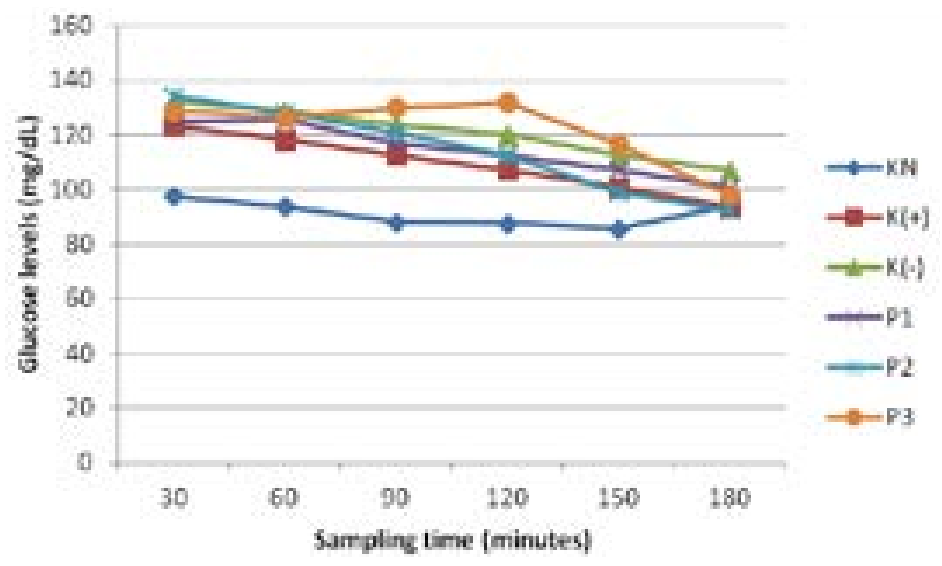

Figure 1. Decrease in Blood Glucose Level During Observation Time

$\mathrm{KN}=$ normal control, $\mathrm{K}(+)$ = positive control, $\mathrm{K}(-)$ = negative control, $\mathrm{P} 1$ = treated with Kombucha fermented for 8 days, $\mathrm{P} 2=$ treated with Kombucha fermented for 14 days, P3 = treated with Kombucha fermented for 21 days 


\section{Conflict of Interest}

None declared.

\section{References}

1. Srihari T, Karthikesan K, Ashokkumar $\mathrm{N}$, et al. Antihyperglycaemic efficacy of kombucha in streptozotocin-induced rats. Journal of Functional Foods. 2013;5(4):1794-1802.

2. Chakravorty S, Bhattacharya S, Chatzinotas A, et al. Kombucha tea fermentation: Microbial and biochemical dynamics. International Journal of Food Microbial. 2016;220:63-72.

3. Gaggìa $\mathrm{F}$, Baffoni L, Galiano $\mathrm{M}$, et al. Kombucha beverage from green, black and rooibos teas: A comparative study looking at microbiology, chemistry and antioxidant activity. Nutrients. 2018;11(1):1.

4. Defilippis F, Troise AD, Vitaglione $\mathrm{P}$, et al. Different temperatures select distinctive acetic acid bacteria species and promotes organic acids production during Kombucha tea fermentation. Food Microbiology. 2018;73:11-16.

5. Jayabalan R, Malbasa RV, LoncarES, etal. A review on kombucha teamicrobiology, composition, fermentation, beneficial effects, toxicity, and tea tungus. Comprehensive Reviews in Food Science and Food Safety. 2014;13:538- 550.

6. Nguyen NK, Dong NT, Nguyen HT, et al. Lactic acid bacteria: promising supplements for enhancing the biological activities of kombucha. Springerplus. 2015;4:91.

7. Barati F, Javanbakht J, Adib-Hashemi $\mathrm{F}$, et al. Histopathological and clinical evaluation of Kombucha tea and Nitrofurazone on cutaneous full-thickness wounds healing in rats: An experimental study. Pathology. 2016;11(1):117.

8. Johnson MH, Demejia EG. Phenolic compounds from fermented berry beverages modulated gene and protein expression to increase insulin secretion from pancreatic $\beta$-cells in vitro. Journal of Agriculture and Food Chemistry 64(12): 2569-2581.

9. Zubaidah E, Apriyadi TE, Kalsum U, et al. In vivo evaluation of snake fruit Kombucha as hyperglycemia therapeutic agent. International Food Research Journal. 2018;25(1):453-457.

10. Aloulou A, Hamden K, Elloumi D, et al. Hypoglycemic and antilipidemic properties of Kombucha tea in alloxan-induced diabetic rats. $B M C$ Complementary and Alternative Medicine. 2012;12: 63-71.

11. Katarzyna NS, Barbara S, Iwona S, et al. Acid contents and the effect of fermentation condition of Kombucha tea beverages on physicochemical, microbiological and sensory properties CyTA Journal of Food. 2017;15:4601607 , 